\title{
Qualidade de mudas de Moringa oleifera Lam. cultivadas em substratos com fibra de coco verde e compostos orgânicos
}

\author{
Luciana Aparecida Rodrigues ${ }^{*}$, Tiago Araujo Muniz ${ }^{2}$, Solange Silva Samarão ${ }^{3}$, André Erse Cyrino
}

10.1590/0034-737X201663040016

\section{RESUMO}

A Moringa oleifera Lam. (moringa) é uma espécie vegetal com grande potencial de uso nos setores alimentício, medicinal e forrageiro, no processo de clareamento e descontaminação de água, produção de biocombustível, dentre outros. Técnicas de produção de mudas com uso de resíduos regionais são apropriadas para a introdução desta espécie em comunidades carentes. O objetivo deste trabalho foi avaliar as respostas nutricionais e de crescimento de mudas de moringa, cultivadas em substratos com diferentes concentrações de fibra da casca de coco verde (FCV), associadas a composto orgânico (CO). O delineamento experimental adotado foi em blocos casualizados. Foram testados dois compostos orgânicos: o composto de lixo urbano (CLU) e o vermicomposto (VC) associados a FCV em várias proporções volumétricas (CO:FCV): 0:100; 25:75; 50:50; 75:25 a 100:0\%. Após 51 dias de cultivo, foi observado que o aumento da concentração de FCV em relação ao composto orgânico reduziu a altura, a massa da matéria seca, o Índice de Qualidade de Dickson e os conteúdos de N, P, K, Ca e Mg das mudas. Não houve diferença, para essas características comparandose o uso do composto de lixo urbano e o do vermicomposto. Substratos com 100\% de FCV e sem adubação complementar ocasionaram sintomas de deficiência de $\mathrm{N}$ nas mudas de moringa.

Palavras-chave: moringa; composto de lixo urbano; vermicomposto; crescimento vegetal.

\section{ABSTRACT}

\section{Quality of Moringa oleifera Lam. seedlings cultivated in substrates with green coconut fiber and organic compounds}

Moringa oleifera Lam. is a vegetal species with great potential to use as food, medicine and forage as well as water clarifying and decontamination agent, biofuel, among others. Low cost techniques for seedling production using regional residues are useful to introduce this species in low-income communities. This research aimed to evaluate the nutritional responses and growth of moringa seedlings cultivated in substrates with different concentrations of green coconut fiber (GCF) associated to organic compounds (OC). The experiment was performed in randomized blocks. Two organic compounds were tested: the urban waste compost (UWC) and earthworm compost (EC) associated with GCF in various volumetric proportions (OC: GCF): $0: 100 ; 25: 75 ; 50: 50 ; 75: 25$ and 100:0\%. The data after 51 days of cultivation showed that the increase of the concentration of GCF in relation to the organic compound decreased height, dry biomass, Dickson Quality Index and the contents of N, P, K, Ca and Mg of the seedlings. There was no difference for these characteristics between the use of UWC and the EC. The substrate with $100 \%$ of GCF without complementary fertilizers caused symptoms of $\mathrm{N}$ deficiency in the moringa seedlings.

Key words: Moringa; urban solid waste compost; earthworm compost; vegetable growth.

\footnotetext{
Submetido em 17/09/2014 e aprovado em 15/01/2016.

'Universidade Estadual do Norte Fluminense Darcy Ribeiro, Laboratório de Solos, Campos dos Goytacazes, Rio de Janeiro, Brasil. lua@uenf.br; lucianaaparecidarodrigues@gmail.com ${ }^{2}$ Fundação de Apoio a Escola Técnica, Campos dos Goytacazes, Rio de Janeiro, Brasil. tiagoamuniz@yahoo.com.br

${ }^{3}$ Universidade Estadual do Norte Fluminense Darcy Ribeiro, Laboratório de Sanidade Animal, Campos dos Goytacazes, Rio de Janeiro, Brasil. solangesamarao@gmail.com.br

${ }^{4}$ Núcleo de Defesa agropecuária de Itaocara, Itaocara, Rio de Janeiro, Brasil andreerse@ig.com.br

*Autora para correspondência: lua@uenf.br
} 


\section{INTRODUÇÃO}

A Moringa oleifera Lam. (moringa) é uma espécie leguminosa perene e arbórea, da família Moringaceae, originária do Noroeste Indiano, distribuída pela Índia, Egito, Filipinas, Ceilão, Tailândia, Malásia, Burma, Paquistão, Singapura, Jamaica e Nigéria (Corrêa, 1984). No Brasil os primeiros registros de frutos obtidos pelo plantio da moringa foram do Jardim Botânico do Rio de Janeiro, em seguida foram disseminadas principalmente na região nordeste, em regiões áridas e semiáridas, as quais possuem precipitações anuais abaixo de $300 \mathrm{~mm}$, onde é conhecida como Lírio Branco ou Acácia-Branca, no entanto, pode ser encontrada em praticamente todo o território brasileiro, devido a ampla divulgação dos seus usos e das campanhas de doação de sementes (Siguemoto, 2013) .

De acordo com Ferreira et al. (2008), vários são os usos da moringa: como forrageira (folhas, frutos e sementes); medicinal (todas as partes da planta); condimentar (principalmente as raízes); culinário; na indústria de cosméticos (óleo extraído das sementes); melífero (flores); e como combustível (madeira e óleo). No Brasil, há um esforço no sentido de difundir o cultivo dessa hortaliça arbórea caracterizada por ser rica em vitamina $\mathrm{A}$, apresentar nas folhas saponinas, fitatos e taninos, com baixos fatores antinutricionais.

As sementes de moringa apresentam cerca de $40 \%$ de óleo, sendo que $78 \%$ deste é ácido oleico, o que o torna potencial para uso como biodiesel (Answar \& Bhanger, 2003). Elas apresentam glucosinolatos, fitatos e atividade hemaglutinante (Ferreira et al., 2008). A solução coagulante obtida das sementes de moringa é eficiente para proporcionar o clareamento de águas com turbidez variando de 50 até 100 NTU (Unidades Nefelométrica de Turbidez) (Paterniani et al., 2009). Sengupta et al. (2012) observaram a redução de 94 a $98 \%$ dos ovos de helmintos em animais tratados com extrato da semente de moringa. Amaral et al. (2006) observaram que a eliminação de mais de $80 \%$ de Escherichia coli, em amostras de água turvas e contaminadas, foi obtida pelo efeito do extrato da semente de moringa na sedimentação dos sólidos em suspensão na água, associado ao da exposição da água à radiação solar.

Dada a multiplicidade de seus usos, a moringa vem sendo cultivada no mundo, e os incentivos aos plantios vêm aumentando. Nas zonas rurais do nordeste brasileiro, a utilização das sementes de moringa no tratamento de água para o consumo humano tem sido prática frequente (Ferreira et al., 2008), tendo em vista ser uma alternativa de fácil acesso e de baixo custo e, também, ser facilmente empregada nas regiões onde o acesso à água para fins agrícolas é escasso, especialmente em épocas de seca, quando a agricultura é muito prejudicada por esse fator. As comunidades rurais, mais particularmente os assenta- mentos instalados no norte Fluminense, apresentam grande número de pessoas que utilizam água de poços, rios ou lagoas, sem quaisquer tratamentos. $\mathrm{O}$ incentivo aos plantios de moringa nestas áreas justifica-se, não somente pela possibilidade do uso de suas sementes no processo de tratamento da água e potencial de produção de biodiesel, mas também de uso das folhas na alimentação humana e animal. Plantios em maior escala requerem produção de mudas de boa qualidade.

O substrato a ser utilizado na produção das mudas necessita ser composto por matéria-prima de fácil aquisição, de baixo custo e de material de baixa densidade (leve), para facilitar o transporte das mudas para o campo, e de constante disponibilidade. Os resíduos da agroindústria ou do lixo orgânico urbano apresentam potencial para essa utilização (Coutinho et al., 2006), além de esse uso auxiliar na minimização da poluição decorrente do acúmulo desse resíduo no ambiente.

Um bom substrato deve apresentar características físicas e químicas adequadas à espécie em questão; o que dificilmente se encontrará em um único material. O substrato deve apresentar grau avançado de estabilidade química e biológica, sendo a forma mais eficiente de atingir essa característica os processos da compostagem ou da vermicompostagem dos resíduos orgânicos. Os compostos orgânicos têm apresentado respostas promissoras para uso nas produções agrícola e florestal, com respostas positivas quanto ao crescimento e à nutrição mineral das plantas (Barichello et al., 2001; Veras, 2004; Coutinho et al., 2006; Loureiro et al., 2007; Nóbrega et al., 2008a; Nóbrega et al., 2008b).

O composto resultante da compostagem ou da vermicompostagem apresenta condições favoráveis ao crescimento das plantas, pela estabilidade dos processos microbianos, boa aeração, estrutura, capacidade de retenção de água e capacidade de regulação da temperatura do solo, além do fornecimento de nutrientes, que podem estar prontamente disponíveis ou complexados (Trindade et al., 2001). O vermicomposto é conhecido por seus altos níveis de fosfato, nitrogênio e outros nutrientes, e também contêm polissacarídeos que agregam partículas do solo (Gliessman, 2001). De acordo com Canellas et al. (2001; 2006), as respostas positivas dos compostos devem-se, ainda, à grande quantidade de ácidos húmicos que apresentam influência direta sobre a planta ou na alteração das características químicas, físicas e biológicas do substrato.

A fibra de coco verde é outro material alternativo para a produção de substratos (Noguera et al., 2000; Carrijo et al., 2002; Lacerda et al., 2006). O alto potencial poluidor do coco verde, proveniente da exploração comercial da água de coco, tem trazido grandes problemas para as áreas urbanas, sendo necessário ampliar e incentivar formas de 
sua reutilização. A proporção de fibras na composição do substrato deve ser avaliada com critério, para proporcionar ao substrato as características químicas e físicas ideais para cada espécie a ser cultivada (Bezerra \& Rosa, 2002; Yamakami et al., 2006; Costa et al., 2007). A fibra de coco apresenta alta relação $\mathrm{C} / \mathrm{N}$ e alta porosidade (Bezerra \& Rosa, 2002), requerendo sua associação com materiais com maiores teores de $\mathrm{N}$ e de maior densidade. Bezerra et al. (2004) observaram que o pó de coco lavado, misturado a solo e húmus de minhoca aumentou a percentagem e a velocidade de germinação de sementes de moringa, mais que o uso de vermiculita. Assim, o uso da fibra do coco, do composto de lixo urbano e do vermicomposto, como substrato para produção de mudas, pode transformar materiais com alto potencial poluidor em subprodutos com valor no mercado ou na produção de mudas de espécies florestais importantes para o uso em comunidades carentes, como é o caso da moringa.

Diante do exposto, o objetivo deste trabalho foi avaliar as respostas nutricionais e de crescimento de mudas de moringa cultivadas em substrato com diferentes misturas de fibra da casca de coco verde, associada ao composto de lixo urbano ou ao vermicomposto.

\section{MATERIAL E MÉTODOS}

O experimento foi conduzido em casa de vegetação, localizada em Campos dos Goytacazes, RJ, de 13 de junho de 2007 a 31 de agosto de 2007. Os valores de temperatura e umidade relativa do ar, obtidos pela miniestação meteorológica WhatchDog 2900ET instalada dentro da casa de vegetação, variaram de 15 a $25^{\circ} \mathrm{C}$ e de 60 a $80 \%$, respectivamente.

O delineamento experimental adotado foi em blocos casualizados com 5 repetições. Os tratamentos constaram da produção de substratos a partir da mistura de composto orgânico (CO) com a fibra de coco verde (FCV), em cinco proporções. Foram testados dois $\mathrm{CO}$, o composto de lixo urbano (CLU) e o vermicomposto (VC), resultando em dez substratos. As proporções CO:FVC foram: 0:100; 25:75; 50:50; 75:25 e 100:0\% com base no volume final do substrato. Os valores em massa (g) das proporções CO:FVC 0:100; 25:75; 50:50; 75:25 e 100:0\% foram, respectivamente, $0,0: 50 ; 286,3: 37,5 ; 671,0: 25,0$; 12,5:909,0; 1145,0:0,0 g.

A FCV foi obtida mediante a coleta de cascas de coco de comerciantes de água de coco do Município de Campos dos Goytacazes, RJ. A casca de coco verde foi cortada com machado, e triturada, em seguida, por uma máquina trituradora de forragem, resultando em fibras de comprimento médio de $17 \mathrm{~cm}$. As fibras foram colocadas para secar ao ar e à sombra por 30 dias, quando foi realizada sua análise química (Tabela 1).
O CLU foi obtido a partir do processo de compostagem do lixo orgânico, coletado no município de Miracema, RJ. O CLU foi considerado apropriado, após a estabilização da temperatura e do $\mathrm{pH}$ e depois de a cor do material ficar escura e sem odor de substâncias em estado de putrefação. O VC foi obtido por meio do processo de vermicompostagem, tendo como substrato o próprio lixo urbano já compostado. Foram realizadas análises químicas do CLU e do VC (Tabela 2).

As sementes da moringa foram obtidas junto à Embrapa Tabuleiros Costeiros, em Aracaju, SE. A semeadura foi realizada em copos plásticos descartáveis $(100 \mathrm{~mL})$, utilizandose $100 \mathrm{~mL}$ de substrato comercial Plantmax Florestais $®$. Foi adicionada uma semente de moringa por copo, sendo essas cobertas por cerca de $20 \mathrm{~mL}$ do mesmo substrato. Aos 19 dias após o semeio, foi medida a altura das plântulas. Logo após, elas foram transplantadas para vasos com $1,0 \mathrm{dm}^{3} \mathrm{dos}$ substratos, correspondendo aos distintos tratamentos.

As mudas foram cultivadas, durante 51 dias, sendo irrigadas diariamente, conforme a necessidade, e avaliadas, semanalmente, quanto à altura (com régua milimetrada, medindo-se a haste principal da planta, da base da planta rente ao substrato até a brotação terminal da haste principal da planta) e quanto ao diâmetro do coleto (tomando-se as medidas na base do coleto, a cerca de $1 \mathrm{~cm}$ do substrato, com paquímetro digital).

$\mathrm{Na}$ avaliação final, determinou-se a matéria seca das plantas. Para tanto, a raiz secundária tuberosa de cada planta foi lavada com jatos de água sob peneira de 2,0 mm. A parte aérea e as raízes tuberosas secundárias foram separadamente acondicionadas em sacos de papel, secadas individualmente em estufa de circulação forçada de ar, a temperatura de $65^{\circ} \mathrm{C}$, por 72 horas, e avaliadas quanto à matéria seca. O material seco foi triturado (em moinho tipo Willey) e peneirado (em peneira de 20 malhas por polegada) e armazenado em frascos hermeticamente fechados. Esse material foi submetido à oxidação por digestão nítricoperclórica (na proporção de 4:1), determinando-se o $\mathrm{P}$, por colorimetria; o K, por fotometria de chama; $\mathrm{Ca}$ e $\mathrm{Mg}$ por espectrofotometria de absorção atômica e o N-total, após digestão sulfúrica, pelo método de Kjeldahl (Malavolta et al., 1997). Foi calculado o conteúdo de cada nutriente com base no teor foliar e na matéria seca da planta.

O Índice de Qualidade de Dickson (IQD) (Dickson et al., 1960) foi determinado pela seguinte fórmula:

$$
I Q D=\frac{M S T}{\left[\left(\frac{H}{D C}\right)+\left(\frac{M S P A}{M S R}\right)\right]}
$$

Em que MST é a matéria seca total (g), H é a altura da planta $(\mathrm{cm})$, DC é o diâmetro do coleto $(\mathrm{mm})$, MSPA é a matéria seca da parte aérea (g) e MSR é a matéria seca da $\operatorname{raiz}(\mathrm{g})$. 
Luciana Aparecida Rodrigues et al.

Tabela 1: Propriedades físicas e químicas do substrato de fibra da casca de coco verde

\begin{tabular}{|c|c|c|c|c|c|c|c|c|c|c|c|}
\hline \multirow[t]{2}{*}{ pH } & C.E & $\mathbf{K}^{+}$ & $\mathrm{Na}^{+}$ & $\mathrm{Ca}^{++}$ & $\mathbf{M g}^{++}$ & $\mathrm{Cu}$ & Zn & Mn & Cl- & $\mathbf{P}^{*}$ & $\mathbf{R A} \mathbf{S}^{\text {** }}$ \\
\hline & $\mathrm{mmhos} / \mathrm{cm}$ & \multicolumn{10}{|c|}{$\mathrm{mg} / \mathrm{dm}^{3}$} \\
\hline 5,5 & 2,31 & 1080,7 & 511,06 & 12,22 & 26,52 & 0,18 & 0,32 & 0,13 & 1704,0 & 48,0 & 20,41 \\
\hline
\end{tabular}

Extração pelo Método de Saturação - 45,84g de Fibra + 400 ml H2O. P* - Método Solo (Extrator Carolina do Norte), pH em água.

** - Relação de adsorção de sódio.

Os dados experimentais foram submetidos à análise de variância (ANOVA) por meio do programa computacional SAEG, versão 9.1 (Universidade Federal de Viçosa, 2007). Os compostos orgânicos VC e CLU foram comparados pelo teste de $\mathrm{F}$ a 5\% de significância. O efeito da percentagem do $\mathrm{CO}$ e da FCV foram analisados pelo teste de Tukey até 5\% de significância. Para a altura das mudas em cada substrato, também foram selecionados modelos de regressão em função do tempo de cultivo nos vasos.

\section{RESULTADOS E DISCUSSÃO}

Não foram verificadas diferenças significativas entre os compostos orgânicos testados (CLU e VC) para as características de crescimento das mudas de moringa, mas houve efeito significativo da percentagem de $\mathrm{CO}$ na composição dos substratos para essas características. Proporções crescentes de CO (tanto o VC quanto o CLU) aumentaram a matéria seca da parte aérea, a matéria seca das raízes tuberosas, a matéria seca total, o diâmetro do coleto, a altura e o IQD das mudas de moringa na avaliação aos 51 dias após o transplantio para os vasos (Tabela 3).

$\mathrm{O}$ percentual de redução das mudas de moringa entre os substratos com 100 e $0 \%$ de CO foi de $94 ; 86 ; 89 ; 54 ; 47$ e $80 \%$, respectivamente, para matéria seca da parte aérea, matéria seca da raiz, matéria seca total, diâmetro, altura e IQD (Tabela 3). A variável de crescimento mais sensível ao incremento da quantidade do $\mathrm{CO}$ na composição do substrato foi a massa seca da parte aérea.

Os valores do IQD variaram entre 5,9 e 7,3 para os substratos com 0 e com $100 \%$ de CO, respectivamente. Esses valores encontram-se acima do que é observado para algumas espécies arbóreas. Da Silva et al. (2012) observaram valores do IQD de, no máximo, 0,19, em mudas de eucalipto (clone de Eucalyptus urophylla x E. grandis) com 90 dias, cultivadas em substrato com fibra de coco + Vermiculita (1:1). Em mogno africano (Khaya anthotheca Welv.) cultivado em areia irrigada com solução nutritiva completa, com omissão de $\mathrm{P}$ e com omissão de $\mathrm{N}$, os valores do IQD foram de 0,$87 ; 0,43$ e 1,01 , respectivamente (Vieira et al., 2014). IQD maiores que os observados neste experimento foram obtidos em mudas de Cedrela fissilis Vell., cultivadas em diferentes substratos, que apresentaram, em média, o valor de 10,3 (Oliveira et al., 2014). Neste experimento, a baixa relação matéria seca da parte aérea/ matéria seca das raízes influenciou nos valores do IQD. Os valores da matéria seca das raízes (raiz tuberosa) foram pelo menos 2,5 vezes maiores que os valores da matéria seca da parte aérea.

Foram selecionadas regressões polinomiais para altura das plantas em função do tempo de cultivo, com as médias dos valores de altura com ambos os compostos. As respostas do crescimento das mudas em altura, nesse período, foram crescentes e lineares (Figura 1). Verificouse que, quanto maior o percentual de $\mathrm{CO}$ na composição do substrato, maior foi o crescimento das mudas em altura. Aos 51 dias, a altura das mudas atingiu valores máximos estimados de 31,$4 ; 28,4 ; 27,4 ; 18,5$ e $13,0 \mathrm{~cm}$, respectivamente, para os substratos com mistura CO:FCV de 0:100; 75:25; 50:50; $25: 75$ e 100:0\% na sua composição. De acordo com Carneiro (1995), a altura das mudas na ocasião do plantio no campo exerce um papel importante na sobrevivência e no desenvolvimento nos primeiros anos após o plantio. Há limites no crescimento em altura das mudas no viveiro, acima e abaixo dos quais, o desenvolvimento não é satisfatório no campo. Além disso, a maior taxa de cresci-

Tabela 2: Propriedades química do composto de lixo urbano (CLU) e vermicomposto (VC)

\begin{tabular}{lcrc}
\hline \multirow{2}{*}{ Análise } & Unidades & \multicolumn{2}{c}{ Composto Orgânico } \\
\cline { 3 - 4 } & & CLU & VC \\
\hline $\mathrm{pH}$ & - & 7,70 & 7,80 \\
$\mathrm{P} *$ & $\mathrm{mg} / \mathrm{dm}^{3}$ & 462,00 & 528,00 \\
$\mathrm{~K}^{*}$ & $\mathrm{mg} / \mathrm{dm}^{3}$ & 2366,00 & 2782,00 \\
$\mathrm{Ca}$ & $\mathrm{cmol}_{\mathrm{c}} / \mathrm{dm}^{3}$ & 20,00 & 15,30 \\
$\mathrm{Mg}$ & $\mathrm{cmol}_{\mathrm{c}} / \mathrm{dm}^{3}$ & 5,30 & 4,80 \\
$\mathrm{Al}$ & $\mathrm{cmol}_{\mathrm{c}} / \mathrm{dm}^{3}$ & 0,00 & 0,00 \\
$\mathrm{H}+\mathrm{Al}$ & $\mathrm{cmol} / \mathrm{dm}^{3}$ & 1,40 & 1,30 \\
$\mathrm{Na}$ & $\mathrm{cmol} / \mathrm{dm}^{3}$ & 4,07 & 4,68 \\
$\mathrm{C}$ & $\%$ & 5,50 & 3,900 \\
$\mathrm{MO}$ & $\mathrm{g} / \mathrm{dm}^{3}$ & 94,80 & 67,20 \\
$\mathrm{~T}$ & $\mathrm{cmol} / \mathrm{dm}^{3}$ & 36,80 & 33,20 \\
$\mathrm{t}$ & $\mathrm{cmol} / \mathrm{dm}^{3}$ & 35,40 & 31,90 \\
$\mathrm{~V}$ & $\%$ & 96,00 & 96,00 \\
$\mathrm{Fe}$ & $\mathrm{mg} / \mathrm{dm}^{3}$ & 31,60 & 44,70 \\
$\mathrm{Cu}$ & $\mathrm{mg} / \mathrm{dm}^{3}$ & 1,90 & 3,00 \\
$\mathrm{Zn}$ & $\mathrm{mg} / \mathrm{dm}^{3}$ & 56,40 & 62,40 \\
$\mathrm{Mn}$ & $\mathrm{mg} / \mathrm{dm}^{3}$ & 82,20 & 61,80 \\
$\mathrm{~S}$ & $\mathrm{mg} / \mathrm{dm}^{3}$ & 7,90 & 5,80 \\
\hline
\end{tabular}

* Extrator Carolina do Norte, $\mathrm{MO}=$ Matéria Orgânica, S.B. = Soma de Bases, $\mathrm{T}=\mathrm{CTC}$ a pH 7,0 , $\mathrm{t}=\mathrm{CTC}$ efetiva, $\mathrm{m}=$ Saturação de Alumínio, $\mathrm{V}=$ Saturação de Base. 
mento das mudas reduz o tempo das mudas no viveiro diminuindo os custos de produção das mesmas. No presente trabalho as mudas cultivadas nos substratos com 50,75 e $100 \%$ de CO, atingiram altura de $20 \mathrm{~cm}$ com menos de 25 dias de cultivo, enquanto nos substratos com somente 25 e $0 \%$ de CO essa altura não foi atingida no tempo de cultivo avaliado, indicando que substratos com mais de $50 \%$ de CO são mais indicados para o crescimento das mudas de moringa e que a FCV prejudica o crescimento inicial das mudas de moringa.

Para os conteúdos de N, P, Ca e Mg da parte aérea das plantas de moringa (Tabela 4) não foram observadas diferenças significativas entre os compostos orgânicos testados para a composição dos substratos. Os conteúdos de $\mathrm{N}, \mathrm{P}, \mathrm{K}, \mathrm{Ca}$ e Mg da parte aérea aumentaram significativamente, com o aumento proporcional do CO na composição do substrato de cultivo (Tabela 4). O aumento observado, entre as concentrações de 0 e de $100 \%$ de $\mathrm{CO}$, no conteúdo da parte aérea de N, P, Ca e Mg foi de 98, 89, 79 e 82\%, respectivamente.

Foram observados sintomas visuais de deficiência de $\mathrm{N}$ no substrato com $100 \%$ de FCV, iniciados aos 35 dias após o transplantio das mudas para os vasos. Esses sintomas foram evidenciados pelo menor crescimento em altura e pela clorose iniciada nas folhas inferiores das plantas que se estendiam em direção às folhas mais jovens. Aos 51 dias após o transplantio, as folhas velhas e a maioria das folhas jovens também apresentavam clorose no substrato com $100 \%$ FCV.

Os sintomas de deficiência nutricional, a redução da matéria seca da parte aérea, das raízes, do IQD (Tabela 3) e do conteúdo de nutrientes da parte aérea das mudas (Tabela 4), observada com o incremento das doses de FCV na composição do substrato ocorreu, provavelmente, por causa dos baixos teores de nutrientes na FCV (Tabela 1) em relação aos do composto orgânico (Tabela 2). Segundo Lima et al. (2011), o composto de lixo urbano propicia o aumento do valor de $\mathrm{pH}$ e dos teores de matéria orgânica, de $\mathrm{P}$, de K, de $\mathrm{Ca}$ e de $\mathrm{Mg}$ no solo, contribuindo para a melhoria das suas características químicas. De acordo com Vieira et al. (2008), a omissão de N, P e Mg da solução nutritiva provocou diminuição da produção de biomassa seca total e favoreceu o crescimento das raízes, aumentando a relação entre raiz e parte aérea, em plantas de moringa. Dentre esses, a omissão do $\mathrm{N}$ foi o que mais levou à diminuição da produção de matéria seca da parte aérea e ao aumento da matéria seca radicular. Bezerra et al. (2004) observaram que a altura, a matéria seca da parte aérea e a matéria seca total de plântulas de moringa foram maiores com o substrato Plantmax $®$ e com a mistura de solo + húmus de minhoca + pó de casca de coco lavado, do que com vermiculita.

Foi observado na FCV alto teor de sódio, o que elevou a condutividade elétrica e também a relação de adsorção de sódio (RAS) (Tabela 1). De acordo com Tomé Jr. (1997), a alta condutividade elétrica proporcionada pelo excesso de sais na zona radicular prejudica a germinação, desenvolvimento e produtividade das plantas. A salinidade prejudica o crescimento inicial das mudas e promove o acúmulo de cátions na parte aérea e nas raízes das plantas. Por outro lado, segundo Freire \& Miranda (2012), na fase inicial de crescimento, as plantas de moringa não inibem a absorção de Na e sua translocação para a parte aérea.

Tabela 3: Crescimento e índice de qualidade de Dickson de mudas de Moringa oleifera LAM aos 51 dias, após o plantio e cultivo em substratos produzidos a partir de diferentes proporções (v/v) de composto orgânico (composto de lixo urbano ou vermicomposto) com fibra de coco verde

\begin{tabular}{|c|c|c|c|c|c|}
\hline \multicolumn{5}{|c|}{ Proporção de Composto orgânico: Fibra de coco verde } & \multirow{2}{*}{$\mathrm{CV}(\%)$} \\
\hline $0: 100$ & $25: 75$ & 50:50 & $75: 25$ & 100:0 & \\
\hline \multicolumn{6}{|c|}{ Matéria seca da parte aérea $\left(\mathrm{g}\right.$ planta $\left.{ }^{-1}\right)$} \\
\hline $0,37 \mathrm{E}$ & $1,08 \mathrm{D}$ & $2,04 \mathrm{C}$ & $3,12 \mathrm{~B}$ & $4,14 \mathrm{~A}$ & 25 \\
\hline \multicolumn{6}{|c|}{ Matéria seca da raiz (g planta ${ }^{-1}$ ) } \\
\hline $1,64 \mathrm{C}$ & $2,38 \mathrm{BC}$ & $6,27 \mathrm{~B}$ & $9,46 \mathrm{~A}$ & $10,60 \mathrm{~A}$ & 37 \\
\hline \multicolumn{6}{|c|}{ Matéria seca total (g planta $\left.{ }^{-1}\right)$} \\
\hline $2,01 \mathrm{C}$ & $4,47 \mathrm{C}$ & $8,32 \mathrm{~B}$ & $12,58 \mathrm{~A}$ & $14,754 \mathrm{~A}$ & 31 \\
\hline \multicolumn{6}{|c|}{ Altura da parte aérea $\left(\mathrm{cm}\right.$ planta $\left.^{-1}\right)$} \\
\hline $12,85 \mathrm{C}$ & $16,60 \mathrm{C}$ & $22,75 \mathrm{~B}$ & $24,65 \mathrm{AB}$ & $28,30 \mathrm{~A}$ & 18 \\
\hline \multicolumn{6}{|c|}{ Diâmetro coleto (mm planta $\left.{ }^{-1}\right)$} \\
\hline $4,43 \mathrm{D}$ & $5,76 \mathrm{CD}$ & $6,90 \mathrm{BC}$ & $7,70 \mathrm{AB}$ & $8,70 \mathrm{~A}$ & 16 \\
\hline \multicolumn{6}{|c|}{ Indice de Qualidade de Dickson } \\
\hline $1,00 \mathrm{C}$ & $2,05 \mathrm{BC}$ & $3,8 \mathrm{~B}$ & $4,44 \mathrm{~A}$ & $5,00 \mathrm{~A}$ & 32 \\
\hline
\end{tabular}

Médias dos valores obtidos para os substratos produzidos com composto de lixo urbano e vermicomposto. Valores seguidos pela mesma letra na linha horizontal não diferem significativamente pelo teste de Tukey $(\mathrm{P}<0,05)$. 


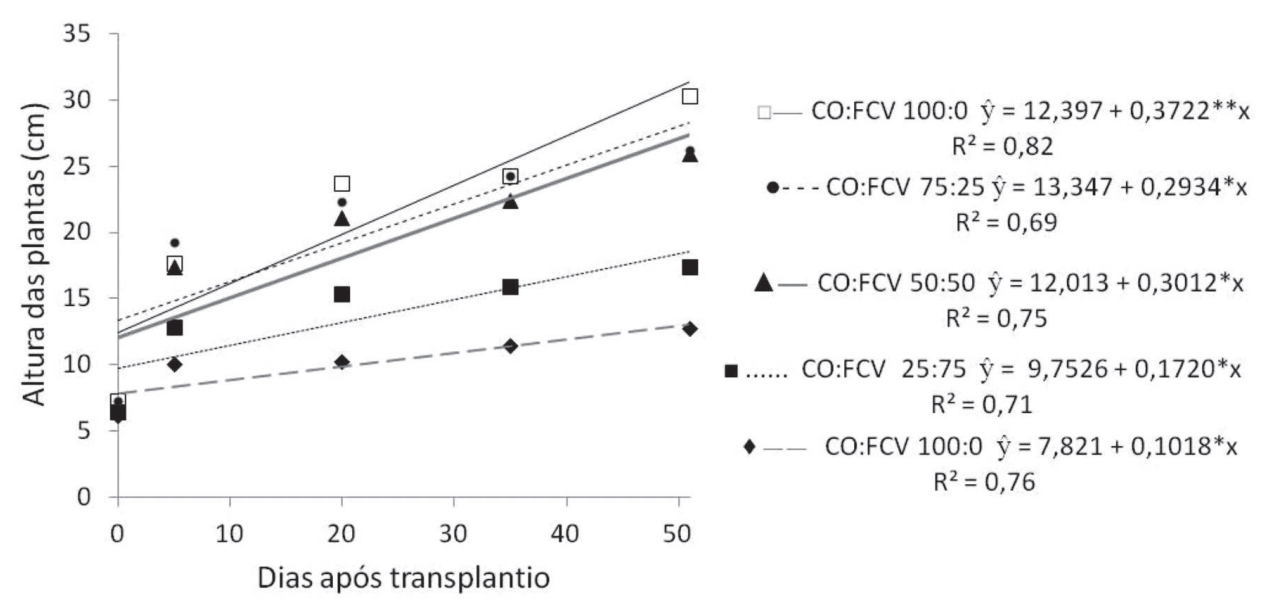

Figura 1: Crescimento em altura de mudas de moringa em função do tempo de cultivo em substratos com diferentes percentagens de fibra de coco verde (FCV) associada a composto orgânico (CO). Média dos valores de composto de lixo urbano e vermicomposto.

Outro problema verificado na composição química da FCV é a relação Ca/Mg que foi de 1/2,2 (Tabela 1), ou seja, na fibra, a quantidade de $\mathrm{Mg}$ é duas vezes maior do que o teor de Ca disponível. Se o equilíbrio Ca:Mg não é adequado, há condições para a deficiência induzida de um dos nutrientes. De acordo com Souza et al. (2007), a relação ideal de $\mathrm{Ca}: \mathrm{Mg}$ no solo varia entre as diferentes culturas, mas os teores de Ca devem ser maiores que o de $\mathrm{Mg}$. Em mudas de angelim pedra, por exemplo, Sena et al. (2010) observaram que a melhor relação Ca:Mg foi de 9:1.

De acordo com Carrijo et al. (2002), para o uso como substrato na produção de mudas, a fibra de coco deve passar por um processo de compostagem. Sem a compostagem, a fibra de coco, em uso como substrato, necessitará ser enriquecida com nutrientes em pré-plantio ou em fertirrigação. A produção das mudas de moringa somente com a FCV, sem os compostos orgânicos, levou a deficiência de nitrogênio, devendo este nutriente ser aplicado antes de 35 dias de cultivo. Para mudas de mangabeira, Dias et al. (2009) recomendam a utilização de proporções de $24 \%$ de esterco, $51 \%$ de terra vegetal, $10 \%$ de fibra de coco, $15 \%$ de areia e $8 \mathrm{~g} \mathrm{dm}^{-3}$ de superfosfato triplo, para obtenção dos máximos teores estimados de macronutrientes e de cobre, no sistema radicular e na parte aérea de mudas. O que foi verificado no presente trabalho é que, para a produção de mudas de moringa, tanto o CLU quanto o $\mathrm{VC}$ foram eficientes em fertilizar o substrato preparado em mistura com FCV, proporcionando respostas semelhantes e não sendo, portanto, necessária a realização da vermicompostagem do CLU. De acordo com Loureiro et al. (2007), a integração dos processos de compostagem e de vermicompostagem produz adubos com características químicas similares, no que se refere à relação $\mathrm{C} / \mathrm{N}$ e aos teores de $\mathrm{Ca}$, $\mathrm{P}$ e $\mathrm{K}$.

Apesar de os maiores incrementos do crescimento e da nutrição das mudas terem ocorrido com os substratos de maior concentração de composto orgânico (100\%), foi observada, na ocasião da colheita, a quebra de raízes e o desmoronamento do torrão no processo de retirada das mudas dos vasos nesses tratamentos. Os substratos com 25 e $50 \%$ de FCV proporcionaram melhor estrutura do torrão, uma vez que as raízes aderiram melhor à $\mathrm{FCV}$, mantendo o sistema radicular mais íntegro, levando a menor quebra de raízes e do torrão (Tabela 3). Estes resultados podem ser importantes no transplantio das mudas para o campo, uma vez que o substrato que proporciona maior proteção ao sistema radicular, evitando o ressecamento e as injúrias mecânicas das raízes. Além disso, a FCV misturada com o composto orgânico torna o substrato mais leve, o que facilitaria o transporte para o campo ou a comercialização desse substrato. Nóbrega et al. (2008), observaram que a proporção de $80 \%$ de composto de lixo e de $20 \%$ de terra de subsolo de Latossolo Vermelho distroférrico, sem calagem, foi a mais adequada para o preparo do substrato, visando à produção de mudas de orelha-demacaco. Já para a produção de mudas de sesbânia, de acordo com Nóbrega et al. (2008b), a máxima produção de matéria seca total, diâmetro do coleto e Índice de Qualidade de Dickson foi com a proporção percentual de solo: CLU de 57: 43.

A FCV, como utilizada neste trabalho, produzida pelo processo de picagem, seguido de trituração em máquina trituradora de forragem com posterior secagem, pode ser utilizada por pequenos produtores rurais, para a produção de mudas de moringa, porém em mistura com o composto orgânico. O uso desses materiais, além de diminuir o custo de produção de mudas tem a vantagem da redução dos problemas ambientais. A utilização de $\mathrm{FCV}$, associada ao CLU ou ao VC, na composição de substrato para produção de mudas de M. oleifera Lam. apresenta potencial de utilização, desde que utilizada em pequenas proporções com CO. 
Tabela 4: Conteúdo de nutrientes em mudas de Moringa oleifera LAM aos 51 dias, após o plantio e cultivo em substratos produzidos a partir de diferentes proporções (v/v) de composto orgânico (composto de lixo urbano ou vermicomposto) com fibra de coco verde

\begin{tabular}{|c|c|c|c|c|c|}
\hline \multicolumn{5}{|c|}{ Proporção de Composto orgânico: Fibra de coco verde } & \multirow{2}{*}{ CV $(\%)$} \\
\hline $0: 100$ & 25:75 & $50: 50$ & $75: 25$ & 100:0 & \\
\hline \multicolumn{6}{|c|}{ Conteúdo N (mg planta $\left.{ }^{-1}\right)$} \\
\hline $5,05 \mathrm{D}$ & $24,23 \mathrm{CD}$ & $51,20 \mathrm{BC}$ & $78,71 \mathrm{~B}$ & $119,95 \mathrm{~A}$ & 27 \\
\hline \multicolumn{6}{|c|}{ Conteúdo P (mg planta $\left.{ }^{-1}\right)$} \\
\hline $0,07 \mathrm{D}$ & $3,17 \mathrm{CD}$ & $5,19 \mathrm{BC}$ & $7,50 \mathrm{AB}$ & $9,86 \mathrm{~A}$ & 20 \\
\hline \multicolumn{6}{|c|}{ Conteúdo $\mathrm{K}\left(\mathrm{mg}\right.$ planta $\left.^{-1}\right)$} \\
\hline $8,71 \mathrm{C}$ & $28,44 \mathrm{C}$ & $66,00 \mathrm{~B}$ & $102,11 \mathrm{~B}$ & $147,68 \mathrm{~A}$ & 22 \\
\hline \multicolumn{6}{|c|}{ Conteúdo $\mathrm{Ca}\left(\mathrm{mg} \mathrm{planta}^{-1}\right)$} \\
\hline $7,83 \mathrm{C}$ & $27,80 \mathrm{BC}$ & $47,68 \mathrm{AB}$ & $48,78 \mathrm{~A}$ & $58,60 \mathrm{~A}$ & 23 \\
\hline \multicolumn{6}{|c|}{ Conteúdo Mg (mg planta ${ }^{-1}$ ) } \\
\hline $1,84 \mathrm{C}$ & $6,20 \mathrm{BC}$ & $9,93 \mathrm{AB}$ & $11,73 \mathrm{~A}$ & $114,66 \mathrm{~A}$ & 25 \\
\hline
\end{tabular}

Médias dos valores obtidos para os substratos produzidos com composto de lixo urbano e vermicomposto. Valores seguidos pela mesma letra na linha horizontal não diferem significativamente pelo teste de Tukey $(\mathrm{P}<0,05)$.

\section{CONCLUSÕES}

O composto de lixo urbano e o vermicomposto proporcionaram respostas iguais de crescimento, de nutrição e de índice de qualidade de Dickson nas plantas de moringa.

O maior crescimento, em altura, matéria seca e conteúdo de nutrientes de plantas de moringa foi obtido em substratos com $100 \%$ de compostos orgânicos.

A fibra de coco verde na composição do substrato deve ser utilizada com cautela e em proporções menores que $25 \%$ uma vez que restringem o crescimento das mudas de moringa e proporcionam sintomas de deficiência nutricional.

\section{AGRADECIMENTOS}

Os autores agradecem à FAPERJ, FENORTE/ TECNORTE e à Prefeitura Municipal de Miracema.

\section{REFERÊNCIAS}

Amaral LA, Rossi Junior OD, Soares E, Barros LS, Lorenzo CS \& Nunes AP (2006) Tratamento alternativo da água utilizando extrato de semente de Moringa oleifera e radiação solar. Arquivo do Instituto Biológico, 73:287-293.

Answar F \& Bhanger MI (2003) Analytical characterization of Moringa oleifera seed oil grown in temperate regions of Pakistan. Journal of Agricultural and Food Chemistry, 22:6558-6563.

Barichello LR, Schumacher MV, Vogel HLM \& Caldeira MVW (2001) Utilização de vermicompoto no crescimento de mudas de Eucalyptus camaldulensis Dehnh. Revista Árvore, 25:397402

Bezerra AME, Moment VG \& Medeiros Filho S (2004) Germinação de sementes e desenvolvimento de plântulas de moringa (Moringa oleifera Lam.) em função do peso da semente e do tipo de substrato. Horticultura Brasileira, 22:295-299.
Bezerra FC \& Rosa MF (2002) Pó da casca de coco verde como substrato para plantas. In: Furlani AMC, Bataglia OC, Abreu CA, Furlani PR, Quaggio JA \& Minami K (Eds.) Caracterização, manejo e qualidade de substratos para produção de plantas. Campinas, IAC. 94p. (Documentos do IAC, 70).

Canellas LP, Santos GA, Rumjanek VM, Moraes AA \& Guridi F (2001) Distribuição da matéria orgânica e características de ácidos húmicos em solos com adição de resíduos de origem urbana. Pesquisa Agropecuária Brasileira, 36:1529-1538.

Canellas LP, Zandonadi DB, Olivares FL \& Façanha AR (2006) Efeitos fisiológicos de substância húmicas. Estímulo às $\mathrm{H}^{+}-$ ATPases. In: Fernandes MS (Ed.) Nutrição Mineral de Plantas. Viçosa, Sociedade Brasileira de Ciência do Solo. p.175-200.

Carneiro JGA (1995) Produção e controle de qualidade de mudas florestais. Curitiba, UFPA/FUPEF; UENF. 451p.

Carrijo AO, Liz RS \& Makishima N (2002) Fibra de coco verde como substrato agrícola. Horticultura Brasileira, 20:533-535.

Corrêa MP (1984) Dicionário das plantas úteis do Brasil e das exóticas cultivadas. Rio de Janeiro, Instituto Brasileiro de Desenvolvimento Florestal. 4324p.

Costa CA, Ramos SJ, Sampaio RA, Guilherme DO \& Fernandes LA (2007) Fibra de coco e resíduo de algodão para substrato de mudas de tomateiro. Horticultura Brasileira, 25:387-391.

Coutinho MP, Carneiro JGA, Barroso DG, Rodrigues LA \& Siqueira J (2006) Substrato de cavas de extração de argila enriquecido com subprodutos agroindustriais e urbanos para produção de mudas de sesbânia. Revista Árvore, 30:147-153.

Da Silva RBG, Simões D \& Da Silva MR (2012) Qualidade de mudas clonais de Eucalyptus urophylla x E. grandis em função do substrato. Revista Brasileira de Engenharia Agrícola e Ambiental, 16:297-302.

Dias TJ, Pereira WE, Cavalcante LF, Sousa VF \& Souza VAB (2009) Mangabeira seedling mineral nutrition cultivated in substrates containing coconut fiber and fertilized with phosphorus. Caatinga, 22:191-201.

Dickson A, Leaf A \& Hosner JF (1960) Quality appraisal of white spruce and white pine seedling stock in nurseries. Forestry Chronicle, 36:10-13.

Rev. Ceres, Viçosa, v. 63, n.4, p. 545-552, jul/ago, 2016 
Ferreira PMP, Farias DF, Oliveira J, Carvalho TA \& Urano AF (2008) Moringa oleifera: bioactive compounds and nutritional potential. Revista de Nutrição. 21:431-437.

Freire ALO \& Miranda JRP (2012) Crescimento e acúmulo de cátions em plantas de moringa mantidas em solos salinos. Pesquisa Florestal, 32:45-51.

Gliessman SR (2001) Agroecologia: processos ecológicos em agricultura sustentável. $2^{\mathrm{a}}$ ed. Porto Alegre, Universidade Federal do Rio Grande do Sul. 653p.

Lacerda MRB, Passos MAA, Rodrigues JJV \& Barreto LP (2006) Características físicas e químicas de substratos à base de pó de coco e resíduo de sisal para produção de mudas de sabiá (Mimosa caesalpiniaefolia Benth). Revista Árvore, 30:163-170.

Lima RLS, Severino LS, Sofiatti V, Gheyi HR \& Arriel NHC (2011) Atributos químicos de substrato de composto de lixo orgânico. Revista Brasileira de Engenharia Agrícola e Ambiental, 15:185192.

Loureiro DC, Aquino AM, Zonta E \& Lima E (2007) Compostagem e vermicompostagem de resíduos domiciliares com esterco bovino para a produção de insumo orgânico. Pesquisa Agropecuária Brasileira, 42:1043-1048.

Malavolta E, Vitti GC \& Oliveira AS (1997) Avaliação do estado nutricional das plantas: princípios e aplicações. $2^{\mathrm{a}}$ ed. Piracicaba, Potafos. 319p.

Nóbrega RSA, Ferreira PAA, Santos JGD, Boas RCV, Nóbrega JCA \& Moreira FMS (2008a) Efeito do composto de lixo urbano e calagem no crescimento inicial de mudas de Enterolobium contortisiliquum (Vell.) Morong. Scientia Forestalis, 3:181-189.

Nóbrega RSA, Paula AMB, Vilas RC, Nóbrega JCA \& Moreira FMS (2008b) Parâmetros morfológicos de mudas de Sesbania virgata (Caz.) Pers e de Anadenanthera peregrina (L.) cultivadas em substrato fertilizado com composto de lixo urbano. Revista Árvore, 32:597-607.

Noguera P, Abad M, Noguera V, Purchades R \& Maquiera A (2000) Coconut coir waste, a new and viable ecologically-friendly pea substitute. Acta Horticulturae, 517:279-286.

Oliveira LR, de Lima SF \& de Lima APL (2014) Crescimento de mudas de cedro-rosa em diferentes substratos. Pesquisa Florestal Brasileira, 34:187-195.

Paterniani JES, Mantovani MC \& Sant'anna MR (2009) Uso de sementes de Moringa oleifera para tratamento de águas superficiais. Revista Brasileira de Engenharia Agrícola e Ambiental, 13:765-771.
Sena J, Tucci C, Lima H \& Hara F (2010) Efeito da calagem e da correção dos teores de $\mathrm{Ca}$ e $\mathrm{Mg}$ no solo sobre o crescimento de mudas de angelim pedra (Dinizia excelsa Ducke). Acta Amazonica, 40:309-318.

Sengupta ME, Keraita B, Olsen A, Boateng OK, Thamsborg SM, Pa'1sdo'ttir GR \& Dalsgaard A (2012) Use of Moringa oleifera seed extracts to reduce helminth egg numbers and turbidity in irrigation water. Water Research, 4:3646-3656.

Siguemoto ES (2013) Composição nutricional e propriedades funcionais do murici (Byrsonima crassifolia) e da moringa (Moringa oleifera). Dissertação de mestrado. Universidade de São Paulo, São Paulo. 125p.

Souza DMG, Miranda LN \& Oliveira AS (2007) Acidez do Solo e sua correção. In: Novais RF, Alvarez V VH, Barros NF, Fontes LF, Cantaruti RB \& Neves JCL (Eds.) Fertilidade solo. Viçosa, SBCS. p.133-204.

Tomé Jr JB (1997) Manual para Interpretação de Análise de Solo. Guaíba, Livraria e Editora Agropecuária. 247p.

Trindade AV, Muchovej RMC, Neves JCL \& Barros NB (2001) Crescimento e nutrição de mudas de Eucalyptus grandis em resposta a composto orgânico ou adubação mineral. Revista Ceres, 48:181-194.

Universidade Federal de Viçosa (2007) SAEG: Sistema para Análises Estatísticas e Genéticas. Versão 9.1. Viçosa, Fundação Arthur Bernardes. CD-ROM.

Veras LRV (2004) A vermicompostagem do lodo de lagoas de tratamento de efluentes industriais consorciado com composto de lixo urbano. Engenharia Sanitária Ambiental, 9:218-224.

Vieira CR, Weber OLS \& Scaramuzza JF (2014) Omissão de macronutrientes no desenvolvimento de mudas de mogno africano. Ecologia e Nutrição Florestal, 2:72-83.

Vieira H, Chaves LHG \& Viégas RA (2008) Crescimento inicial de moringa (Moringa oleifera Lam) sob omissão de nutrientes. Caatinga, 21:51-56.

Yamakami JK, Faria RT, Assis AM \& Rego-Oliveira LV (2006) Cultivo de Cattleya Lindley (Orchidaceae) em substratos alternativos ao xaxim. Acta Scientiarum Agronomy, 28:523-526. 